\title{
Silencing of CXCR7 gene represses growth and invasion and induces apoptosis in colorectal cancer through ERK and $\beta$-arrestin pathways
}

\author{
XIN-XIANG LI ${ }^{1,2^{*}}$, HONG-TU ZHENG ${ }^{1,2^{*}}$, LI-YONG HUANG ${ }^{1,2}$, DE-BING SHI ${ }^{1,2}$, \\ JUN-JIE PENG ${ }^{1,2}$, LEI LIANG $^{1,2}$ and SAN-JUN CAI ${ }^{1,2}$ \\ ${ }^{1}$ Department of Colorectal Surgery, Fudan University Shanghai Cancer Center; ${ }^{2}$ Department of Oncology, \\ Shanghai Medical College, Fudan University, Shanghai 200032, P.R. China
}

Received April 1, 2014; Accepted June 27, 2014

DOI: $10.3892 /$ ijo.2014.2547

\begin{abstract}
The CXC chemokine receptor 7 (CXCR7) has been reported to be involved in cell growth, metastasis and apoptosis in certain cancers. However, the function and molecular mechanisms of CXCR7 in human colorectal cancer (CRC) are still undefined. In the present study, sixty-eight cases of CRC tissues and corresponding adjacent non-cancer tissues (ANCT) were collected, and the expression of CXCR7 was assessed using immunohistochemistry (IHC) in biopsy samples. Furthermore, CXCR7 gene was silenced by small hairpin RNA-mediated lentiviral vector (Lv-shCXCR7), by transfection into human CRC cells (SW480 and HT-29). The levels of $\mathrm{p}$-ERK, $\beta$-arrestin, proliferating cell nuclear antigen (PCNA), matrix metallopeptidase-2 (MMP-2) and caspase-3 (CAS-3) were detected by western blotting. Cell proliferative activities and invasive capability were respectively measured by MTT and Transwell assays. Cell apoptosis was analyzed by flow cytometry. The results demonstrated that CXCR7 expression was significantly upregulated in CRC tissues compared with the ANCT (54.4 vs. $36.8 \%, \mathrm{P}=0.041$ ), and correlated with Dukes staging and depth of invasion $(\mathrm{P}=0.007$; $\mathrm{P}=0.002$ ). Silencing of CXCR7 gene suppressed cell proliferation and invasion, and induced cell apoptosis in CRC cells with decreased expression of $\mathrm{p}$-ERK, $\beta$-arrestin, PCNA and MMP-2 but increased expression of CAS-3. The tumor volumes in the SW480 subcutaneous tumor models treated with Lv-shCXCR7 were significantly smaller than those of the negative control $(\mathrm{NC})$ and $\mathrm{PBS}$ groups $(\mathrm{P}<0.01)$. In conclusion, our findings indicate that upregulation of CXCR7 expression is associated with tumor invasion, and silencing of the CXCR7
\end{abstract}

Correspondence to: Professor Xin-Xiang Li, Department of Colorectal Surgery, Fudan University Shanghai Cancer Center, 270 Dong'an Road, Xuhui District, Shanghai 200032, P.R. China

E-mail: lixinxiang2014@163.com

${ }^{*}$ Contributed equally

Key words: CXCR7, colorectal cancer, growth, invasion gene represses the development of CRC cells through ERK and $\beta$-arrestin pathways, suggesting that CXCR7 may serve as a potential therapeutic target for the treatment of CRC.

\section{Introduction}

Colorectal cancer (CRC) is the third commonest cancer and the third leading cause of cancer death among men and women in the Western world (1), with markedly increasing incidence in China in recent years. Though surgical therapy is one of the main means for treatment of solid tumors, CRC patients still have high morbidity and mortality, of which $20 \%$ of cases suffer from metastatic disease and $30 \%$ of ones recur after curative surgery (2). Therefore, new therapeutic strategies such as gene or targeted therapy have attracted mounting attention for treatment of cancer.

Chemokines are a superfamily of small pro-inflammatory chemoattractant cytokines which can bind to specific $G$ protein-coupled seven-span transmembrane receptors (3). In view of the physiological and pathological function, chemokines binding to CXCR7 receptor direct migration and homing of $\mathrm{CXCR}^{+}$hematopoietic stem cells, indicating a crucial role of CXCR7 in retention of stem cell niches (4). Importantly, it has been reported that $\mathrm{CXCR} 7$ is overexpressed in multiple malignancies including renal cell carcinoma (5), hepatocellular carcinoma (6), and glioma cells (7), and functionally promote inflammation and predict tumor migration and metastasis $(8,9)$. Upregulation of chemokine CXCL12 and its receptor CXCR7 not only regulates the development of some diseases including atherosclerosis, autoimmunity and HIV infection (10), but also participates in tumor proliferation, invasion and metastasis in CRC (11), suggesting a potential research value for CXCR7 in cancer. Co-expression of CXCR7 and its related receptor CXCR4 can assist in signaling pathway transduction in cancer cells $(12,13)$, of which $\beta$-arrestin pathway rather than $\mathrm{G}$ protein-coupled receptor may mediate the implication of CXCR7 in the tumorigenesis (14). Thus, intervention of CXCR7 and downstream signaling pathways by some strategies may show promising therapeutic benefits in cancer (15).

It interest to us that CXCR7 is highly expressed and induces metastatic diseases through the interaction with some 
factors (16). It may activate AKT, ERK and STAT3 signaling pathways involved in the progression of malignant tumors (17). However, few studies exist on the molecular regulatory mechanisms of CXCR7 in CRC. In the present study, we investigated the expression of CXCR7 in human CRC, and the effects of CXCR7 knockdown on biological behavior of CRC cells. We hypothesized that CXCR7 might serve as a potential therapeutic target for the treatment of CRC.

\section{Materials and methods}

Materials. The colon cancer cell lines (SW480 and HT-29) used for these experiments were from the Institute of Biochemistry and Cell Biology (Shanghai, China). Lv-shCXCR7, negative control vector and virion-packaging elements were from Genechem (Shanghai, China). The primer of CXCR7 was synthesized by Applied Biosystems (ABI, USA). All antibodies were from Santa Cruz Biotechnology (USA).

Drugs and reagents. Dulbecco's Modified Eagle's medium (DMEM) and fetal bovine serum (FBS) were from Thermo Fisher Scientific Inc. (Waltham, MA, USA); TRIzol reagent and Lipofectamine 2000 were from Invitrogen (Carlsbad, CA, USA); M-MLV reverse transcriptase was from Promega (Madison, WI, USA); SYBR Green Master Mixture was from Takara (Otsu, Japan). ECL-Plus/kit was from GE Healthcare (Piscataway, NJ, USA).

Clinical samples and data. Tissue microarray was prepared for IHC test. Human CRC tissues and the corresponding ANCT were obtained from biopsy prior to chemotherapy in a total of 68 consecutive cases of CRC admitted in our hospital from January 2008 to December 2012. The study was approved by Medical Ethics Committee of Fudan University, and written informed consent was obtained from the patients or their parents before sample collection. Two pathologists independenly reviewed all the cases.

Tissue microarray. The advanced tissue arrayer (ATA-100, Chemicon International, Tamecula, CA, USA) was used to create holes in a recipient paraffin block and to acquire cylindrical core tissue biopsies with a diameter of $1 \mathrm{~mm}$ from the specific areas of the 'donor' block. The tissue core biopsies were transferred to the recipient paraffin block at defined array positions. The tissue microarrays contained tissue samples from 68 formalin-fixed paraffin-embedded cancer specimens with known diagnosis, and corresponding ANCT from these patients. The block was incubated in an oven at $45^{\circ} \mathrm{C}$ for $20 \mathrm{~min}$ to allow complete embedding of the grafted tissue cylinders in the paraffin of the recipient block, and then stored at $4^{\circ} \mathrm{C}$ until microtome sectioning.

Immunohistochemistry. Anti-CXCR7 antibody (Santa Cruz Biotechnology) was used for IHC detection of the expression of CXCR7 protein in tissue microarrays. Tissue microarray sections were processed for IHC analysis of CXCR7 protein as follows: IHC examinations were carried out on $3 \mathrm{~mm}$ thick sections. For anti-CXCR7 IHC, unmasking was performed with $10 \mathrm{mM}$ sodium citrate buffer, $\mathrm{pH} 6.0$, at $90^{\circ} \mathrm{C}$ for $30 \mathrm{~min}$. For anti-CXCR7 IHC, antigen unmasking was not necessary.
Sections were incubated in $0.03 \%$ hydrogen peroxide for $10 \mathrm{~min}$ at room temperature, to remove endogenous peroxidase activity, and then in blocking serum for $30 \mathrm{~min}$ at room temperature. Anti-CXCR7 antibody was used at a dilution of 1:200. The antibody was incubated overnight at $4^{\circ} \mathrm{C}$. Sections were then washed three times for $5 \mathrm{~min}$ in PBS. Non-specific staining was blocked with $0.5 \%$ casein and $5 \%$ normal serum for $30 \mathrm{~min}$ at room temperature. Finally, staining was developed with diaminobenzidine substrate and sections were counterstained with hematoxylin. PBS replaced CXCR7 antibody in negative controls.

Quantification of protein expression. The expression of CXCR7 was semiquantitatively estimated as the total immunostaining scores, which were calculated as the product of a proportion score and an intensity score. The proportion and intensity of the staining was evaluated independently by two observers. The proportion score reflected the fraction of positive staining cells (score $0,<5 \%$; score $1,5-10 \%$; score $2,10-50 \%$; score 3 , $50-75 \%$; score $4,>75 \%$ ), and the intensity score represented the staining intensity (score 0, no staining; score 1, weak positive; score 2 , moderate positive; score 3 , strong positive). Finally, a total expression score was given ranging from 0 to 12 . Based on the analysis in advance, CXCR7 was regarded as negative expression in CRC tissues if the score was $<2$, and positive expression with the score $\geq 2$.

Cell culture and infection. CRC cells were cultured in DMEM medium supplemented with $10 \%$ heat-inactivated FBS, $100 \mathrm{U} / \mathrm{ml}$ of penicillin and $100 \mu \mathrm{g} / \mathrm{ml}$ of streptomycin. They were all placed in a humidified atmosphere containing $5 \% \mathrm{CO}_{2}$ at $37^{\circ} \mathrm{C}$. On the day of transduction, CRC cells were replated at $5 \times 10^{4}$ cells/well in 24 -well plates containing serum-free growth medium with polybrene $(5 \mathrm{mg} / \mathrm{ml})$. At $50 \%$ confluence, cells were transfected with recombinant Lv-shCXCR7 or control virus at the optimal MOI (multiplicity of infection) of 50 , and cultured at $37^{\circ} \mathrm{C}$ and $5 \% \mathrm{CO}_{2}$ for $4 \mathrm{~h}$. Then supernatant was discarded and serum containing growth medium was added. At 4 days of post-transduction, transduction efficiency was measured by the frequency of green fluorescent protein (GFP)-positive cells. Positive stable transfectants were selected and expanded for further study. The clone in which the Lv-shCXCR7 was transfected was named as Lv-shCXCR7 group, the negative control vectors transfected was the NC group, and CRC cells untreated were the control (CON) group.

Quantitative real-time PCR. To quantitatively determine the mRNA expression level of CXCR7 in CRC cell line, real-time PCR was used. Total RNA of each clone was extracted with TRIzol according to the manufacturer's protocol. Reversetranscription was carried out using M-MLV and cDNA amplification was carried out using SYBR Green Master Mix kit according to the manufacturer's protocol. Target genes were amplified using specific oligonucleotide primer and $\beta$-actin gene was used as an endogenous control. The PCR primer sequences were as follows: CXCR7, 5'-CACCGCA TCTCTTCGACTACTCAGATTCAAGAGATCTGAGTAGT CGAAGAGATGCTTTTTTG-3' and 5'-GATCCAAAAAAG CATCTCTTCGACTACTCAGATCTCTTGAATCTGAGTA 
GTCGAAGAGATGC-3'; $\beta$-actin, 5'-CAACGAATTTGG CTACAGCA-3' and 5'-AGGGGTCTACATGGCAACTG-3'. Data were analyzed using the comparative $C t$ method $\left(2^{-\Delta \Delta C t}\right)$. Three separate experiments were performed for each clone.

Western blot assay. CRC cells were harvested and extracted using lysis buffer (Tris-HCl, SDS, mercaptoethanol, glycerol). Cell extracts were boiled for $5 \mathrm{~min}$ in loading buffer and then equal amount of cell extracts were separated on 15\% SDS-PAGE gels. Separated protein bands were transferred into polyvinylidene fluoride (PVDF) membranes and the membranes were blocked in 5\% skim milk powder. The primary antibodies against CXCR7, $\mathrm{p}$-ERK, $\beta$-arrestin, PCNA, MMP-2 and CAS-3 were diluted according to the instructions of antibodies and incubated overnight at $4^{\circ} \mathrm{C}$. Then, horseradish peroxidase-linked secondary antibodies were added at a dilution ratio of 1:1,000, and incubated at room temperature for $2 \mathrm{~h}$. The membranes were washed with PBS three times and the immunoreactive bands were visualized using ECL-PLUS/ Kit according to the kit instructions. The relative protein level in different groups was normalized to $\beta$-actin concentration. Three separate experiments were performed for each clone.

Cell proliferation assay. Cell proliferation was analyzed with the MTT assay. Briefly, cells infected with Lv-shCXCR7 were incubated in 96-well-plates at a density of $1 \times 10^{5}$ cells per well with DEME medium supplemented with $10 \%$ FBS. Cells were treated with $20 \mu \mathrm{l}$ MTT dye, and then incubated with $150 \mu \mathrm{l}$ of DMSO for $5 \mathrm{~min}$. The color reaction was measured at $570 \mathrm{~nm}$ with enzyme immunoassay analyzer (Bio-Rad, USA). The proliferation activity was calculated for each clone.

Transwell invasion assay. Transwell filters were coated with Matrigel (3.9 $\mu \mathrm{g} / \mu \mathrm{l}, 60-80 \mu \mathrm{l})$ on the upper surface of a polycarbonic membrane (diameter $6.5 \mathrm{~mm}$, pore size $8 \mu \mathrm{m}$ ). After incubating at $37^{\circ} \mathrm{C}$ for $30 \mathrm{~min}$, the Matrigel solidified and served as the extracellular matrix for analysis of tumor cell invasion. Harvested cells $\left(1 \times 10^{5}\right)$ in $100 \mu$ l of serum-free DMEM were added into the upper compartment of the chamber. A total of $200 \mu \mathrm{l}$ conditioned medium derived from NIH3T3 cells was used as a source of chemoattractant, and was placed in the bottom compartment of the chamber. After 24 -h incubation at $37^{\circ} \mathrm{C}$ with $5 \% \mathrm{CO}_{2}$, the medium was removed from the upper chamber. The non-invaded cells on the upper side of the chamber were scraped off with a cotton swab. The cells that had migrated from the Matrigel into the pores of the inserted filter were fixed with $100 \%$ methanol, stained with hematoxylin, and mounted and dried at $80^{\circ} \mathrm{C}$ for $30 \mathrm{~min}$. The number of cells invading through the Matrigel was counted in three randomly selected visual fields from the central and peripheral portion of the filter using an inverted microscope (x200 magnification). Each assay was repeated three times.

Cell apoptosis analysis. To detect cell apoptosis, colon cells were trypsinized, washed with cold PBS and re-suspended in binding buffer according to the instruction of the apoptosis kit. FITCAnnexin V and PI were added to the fixed cells for $20 \mathrm{~min}$ in darkness at room temperature. Then, Annexin V binding buffer was added to the mixture before the fluorescence was measured

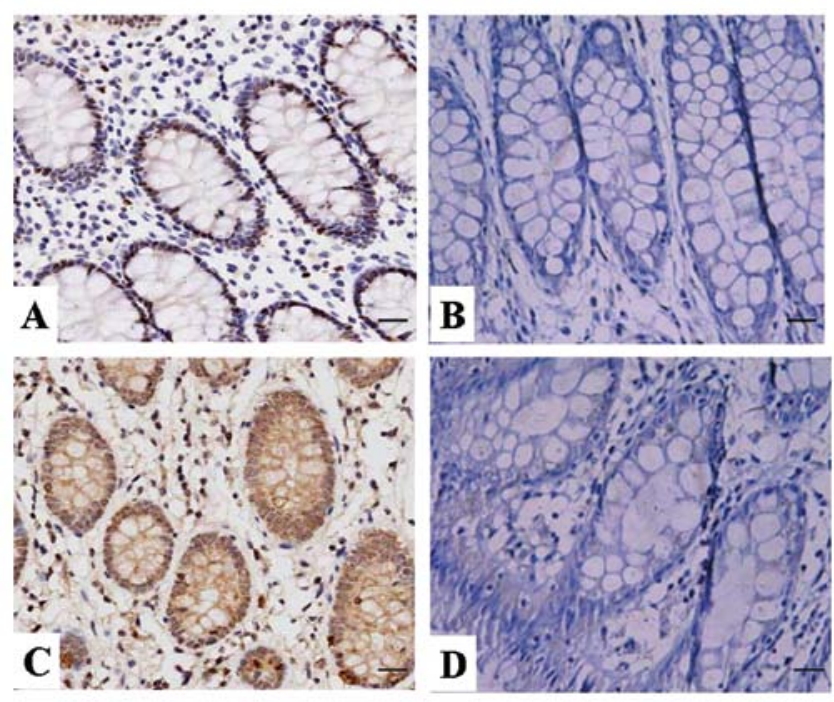

Figure 1. The expression of CXCR7 protein in human CRC (x200). The expression of CXCR7 protein was examined using immunohistochemistry. (A) The positive expression in CRC tissues. (B) The negative expression in CRC tissues. (C) The positive expression in ANCT tissues. (D) The negative expression in ANCT tissues.

on FAC sort flow cytometer. Cell apoptosis was analyzed using the Cell Quest software (Becton-Dickinson, USA). Three separate experiments were performed for each clone.

Subcutaneous tumor model and gene therapy. Six-week-old female immune-deficient nude mice (BALB/c-nu) were bred at the laboratory animal facility. Three mice were injected subcutaneously with $1 \times 10^{7}$ colon cancer cells (SW480) in $50 \mu \mathrm{l}$ of PBS pre-mixed with an equal volume of Matrigel matrix (Becton-Dickinson). Mice were monitored daily and developed a subcutaneous tumor. When the tumor size reached $\sim 5 \mathrm{~mm}$ in length, they were surgically removed, cut into $1-2-\mathrm{mm}^{3}$ pieces, and re-seeded individually into other mice. When tumor size reached $\sim 5 \mathrm{~mm}$ in length, the mice were randomly assigned as PBS group ( $\mathrm{n}=5), \mathrm{NC}$ group $(\mathrm{n}=5)$ and $\mathrm{Lv}$-shCXCR7 group $(\mathrm{n}=5)$. In the treatment group, $15 \mu \mathrm{l}$ of Lv-shCXCR7 was injected into subcutaneous tumors using a multi-site injection format. Injections were repeated every other day after initial treatment. The tumor volume every three days was measured with a caliper, using the formula volume $=(\text { length } \mathrm{x} \text { width })^{2} / 2$.

Statistical analysis. SPSS 20.0 was used for the statistical analysis. Kruskal-Wallis $\mathrm{H}$ test and $\chi^{2}$ test were used to analyze the expression rate in all groups. One-way analysis of variance (ANOVA) was used to analyze the differences between groups. The LSD method of multiple comparisons was used when the probability for ANOVA was statistically significant. Statistical significance was set at $\mathrm{P}<0.05$.

\section{Results}

The expression of $C X C R 7$ protein in human $C R C$. The expression of CXCR7 protein was evaluated using IHC staining. The positive expression of CXCR7 protein was detected in the cytoplasm and cell membrane of CRC tissues and ANCT cells 
Table I. The expression of CXCR7 protein in CRC tissues.

\begin{tabular}{|c|c|c|c|c|c|c|c|c|c|}
\hline \multirow[b]{2}{*}{ Variables } & \multirow[b]{2}{*}{ Group } & \multirow[b]{2}{*}{ Cases $(\mathrm{N})$} & \multicolumn{4}{|c|}{ Expression levels (n) } & \multirow[b]{2}{*}{ Positive rate (\%) } & \multirow[b]{2}{*}{$\chi^{2}$} & \multirow[b]{2}{*}{ P-value } \\
\hline & & & - & + & ++ & +++ & & & \\
\hline \multirow[t]{2}{*}{ CXCR7 } & Cancer & 68 & 31 & 17 & 11 & 9 & 54.4 & 4.165 & 0.041 \\
\hline & ANCT & 68 & 43 & 12 & 8 & 5 & 36.8 & & \\
\hline
\end{tabular}

CRC, colorectal cancer; ANCT, adjacent non-cancer tissue.

Table II. Association between CXCR7 expression and clinicopathologic characteristics of patients with colon cancer.

\begin{tabular}{lccccc}
\hline & \multicolumn{5}{c}{ CXCR7 } \\
& \multicolumn{5}{c}{ expression (n) } \\
\cline { 3 - 4 } Variables & Cases (n) & $(-)$ & $(+)$ & $\chi^{2}$ & P-value \\
& 68 & & & & \\
\hline Age & & 31 & 37 & & \\
$\leq 60$ & 42 & 18 & 24 & & \\
$>60$ & 26 & 13 & 13 & 0.325 & 0.568 \\
Gender & & & & & \\
Male & 35 & 19 & 16 & & \\
Female & 33 & 12 & 21 & 2.167 & 0.141 \\
Tumor size (cm) & & & & & \\
$\leq 5$ & 39 & 16 & 23 & & \\
$>5$ & 29 & 15 & 14 & 0.756 & 0.385 \\
Dukes staging & & & & & \\
A+B & 36 & 22 & 14 & & \\
C+D & 32 & 9 & 23 & 7.322 & 0.007 \\
Depth of invasion & & & & & \\
Within serosa & 41 & 25 & 16 & & \\
Beyond serosa & 27 & 6 & 21 & 9.711 & 0.002 \\
Degree of & & & & & \\
differentiation & & & & & \\
$\quad$ Well & 23 & 12 & 11 & & \\
Moderately & 28 & 11 & 17 & & \\
Poorly & 17 & 8 & 9 & 0.853 & 0.653 \\
CEA(ng/ml) & & & & & \\
$\geq 5$ & 31 & 18 & 13 & & \\
$<5$ & 37 & 13 & 24 & 3.523 & 0.061 \\
\hline$<5$ & & & & \\
\hline
\end{tabular}

(Fig. 1). The positive rates of CXCR7 expression were examined in 54.4\% (37/68) of the CRC tissues, and 36.8\% (25/68) in a small fraction of ANCT. There was a significant difference between them $(\mathrm{P}=0.041$, Table I).

The correlation of CXCR7 expression with clinicopathologic characteristics. The correlation of CXCR7 expression with various clinicopathologic characteristics was analyzed. As shown in Table II, CXCR7 expression did not associate with the age, gender and CEA level of the CRC patients (each
$\mathrm{P}>0.05$ ). Upregulation of CXCR7 expression did not link with tumor size and degree of differentiation $(\mathrm{P}=0.385 ; \mathrm{P}=0.653)$. Moreover, positive expression of CXCR7 was correlated with Dukes staging and depth of invasion $(\mathrm{P}=0.007 ; \mathrm{P}=0.002)$.

Effect of CXCR7 silencing on ERK and $\beta$-arrestin expression. After CRC cells were transfected with Lv-shCXCR7 for $24 \mathrm{~h}$, the effect of CXCR7 on ERK and $\beta$-arrestin expression was identified by real-time PCR and western blot assays, indicating that the expression level of CXCR7 mRNA was significantly knocked down in Lv-shCXCR7 group compared with the NC and $\mathrm{CON}$ groups (each $\left.{ }^{* *} \mathrm{P}<0.01\right)(\mathrm{Fig} .2 \mathrm{~A}$ and $\mathrm{B})$. The expression levels of CXCR7, p-ERK and $\beta$-arrestin proteins were markedly downregulated in Lv-shCXCR7 group in comparison with the $\mathrm{NC}$ and $\mathrm{CON}$ groups (Fig. $2 \mathrm{C}$ and D).

Effect of CXCR7 silencing on cell proliferation. To examine the effect of CXCR7 on cell growth, we investigated the proliferative activities of CRC cells by MTT assay. Silencing of CXCR7 gene could significantly decrease cell proliferative activities of colon cells in a time-dependent manner (Fig. 3A and B). We detected the expression of PCNA by western blotting to determine the effect of CXCR7 on PCNA expression. We found that the amount of PCNA was significantly downregulated in Lv-shCXCR7 group compared with the NC and CON groups (Fig. 3C and D), suggesting that silencing of CXCR7 gene might inhibit CRC cell proliferation through downregulation of the PCNA expression.

Effect of CXCR7 silencing on cell invasion. To determine the effect of CXCR7 on cell invasion, Transwell assay was carried out. The invasive potential was determined on the basis of the ability of cells to invade a matrix barrier containing laminin and type IV collagen, the major components of the basement membrane. Representative micrographs of Transwell filters can be seen in Fig. 4A and B. The invasive potential of CRC cells was significantly reduced in $\mathrm{Lv}$-shCXCR7 group compared to the $\mathrm{NC}$ and CON groups $\left({ }^{* *} \mathrm{P}<0.01\right)$ (Fig. $4 \mathrm{C}$ and $\left.\mathrm{D}\right)$. The endogenous expression of MMP-2, indicated by western blotting, was downregulated in Lv-shCXCR7 group compared with the $\mathrm{NC}$ and $\mathrm{CON}$ groups (Fig. 4E and F), indicating that silencing of CXCR7 gene might inhibit invasive potential of CRC cells through downregulation of MMP-2 expression.

Effect of CXCR7 silencing on cell apoptosis. To determine the effect of CXCR7 on apoptosis of CRC cells, flow cytometric analysis was performed. The apoptotic indexes of CRC cells were much higher in Lv-shCXCR7 group than the 

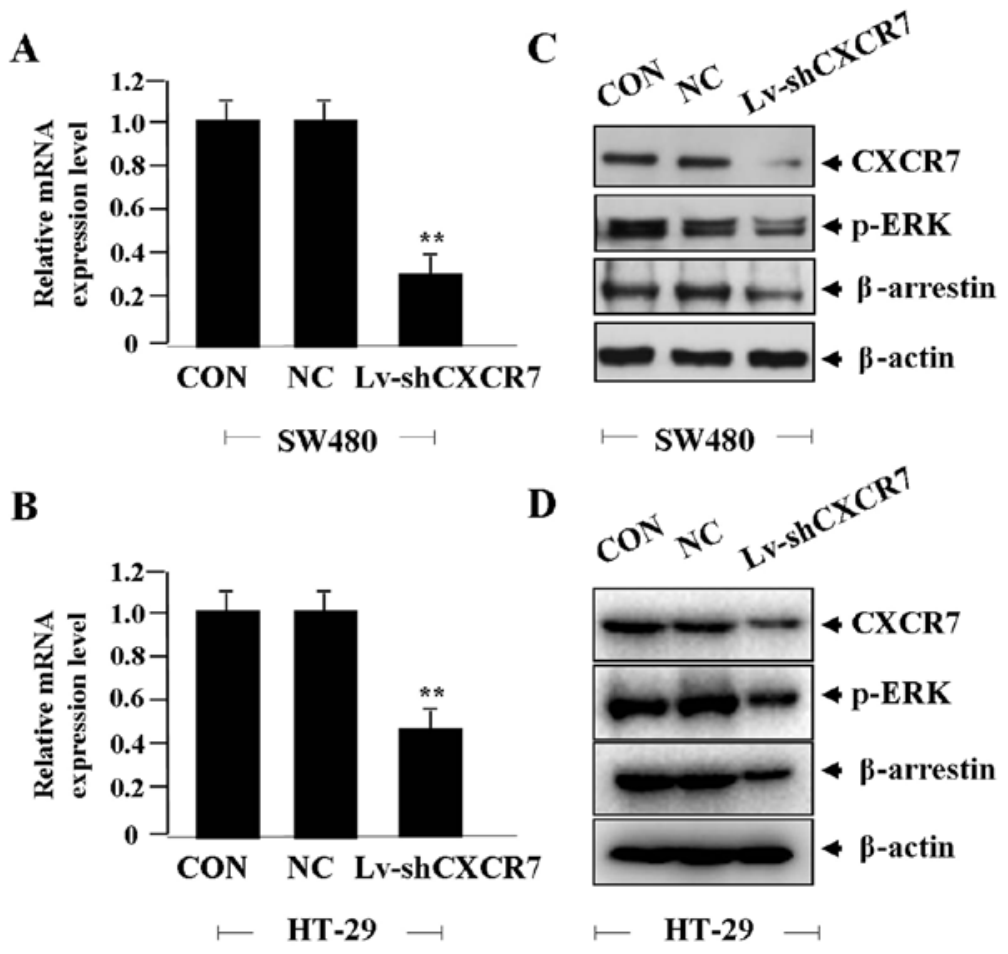

Figure 2. Effect of CXCR7 silencing on p-ERK and $\beta$-arrestin expression. The target gene for CXCR7 and downstream factors were identified by real-time PCR (A and B) and western blot assays (C and D) in CRC cells, indicating that the expression levels of CXCR7, p-ERK and $\beta$-arrestin were significantly downregulated in $\mathrm{Lv}$-shCXCR7 group compared with the CON and NC groups (each ${ }^{* *} \mathrm{P}<0.01$ ).
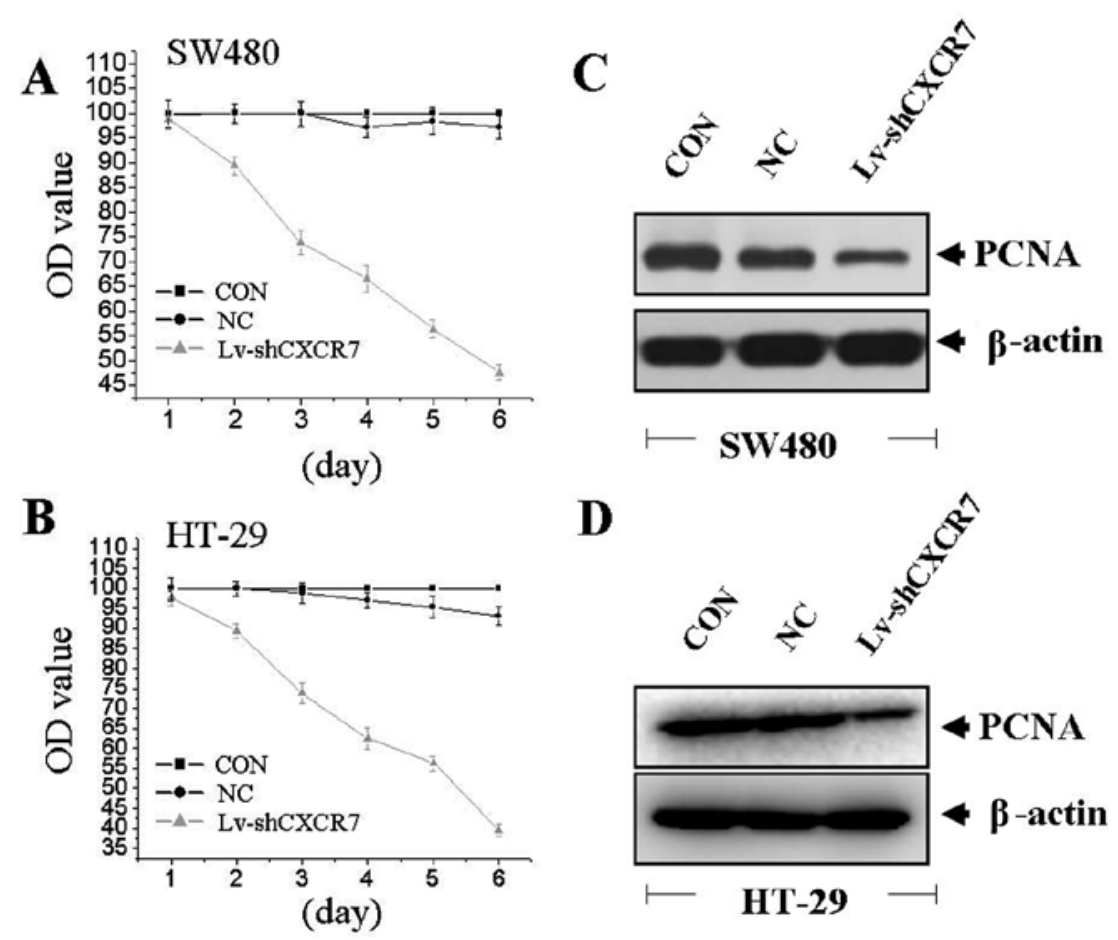

Figure 3. Effect of CXCR7 silencing on cell proliferation. (A and B) The proliferative activities of CRC cells were assessed by MTT assay, indicating that silencing of CXCR7 gene diminished proliferative activities of CRC cells in a time-dependent manner. (C and D) The amount of PCNA protein was decreased in Lv-shCXCR7 group compared with the CON and NC groups.

NC and CON groups ( $\left.{ }^{* *} \mathrm{P}<0.01\right)$ (Fig. 5A-D). The expression of CAS-3 indicated by western blotting, was increased in Lv-shCXCR7 group compared with the NC and CON groups
(Fig. 5E and F), indicating that knockdown of CXCR7 might induce apoptosis of CRC cells through upregulation of CAS-3 expression. 
A

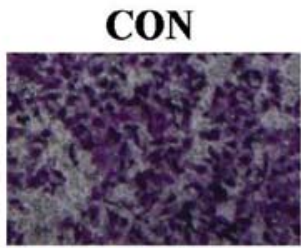

B
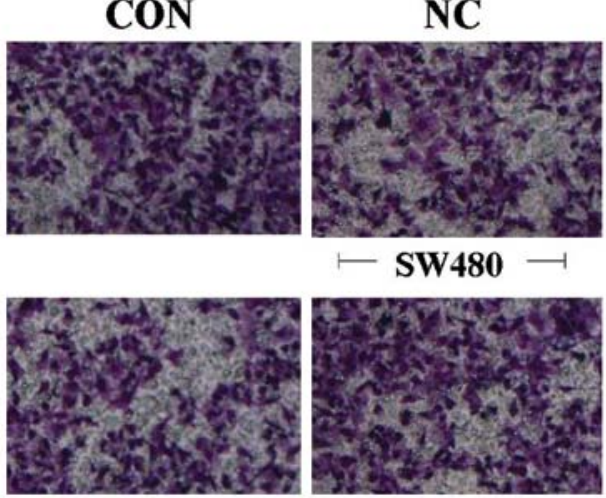

$\longmapsto$ SW480 -

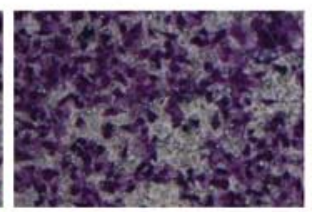

$\longmapsto$ HT-29 $\longrightarrow$
Lv-shCXCR7
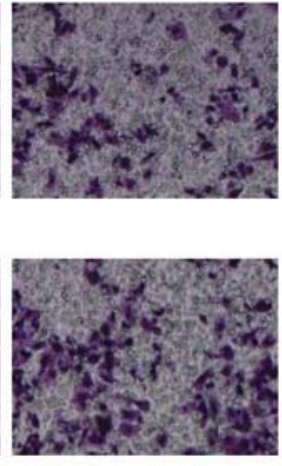

E
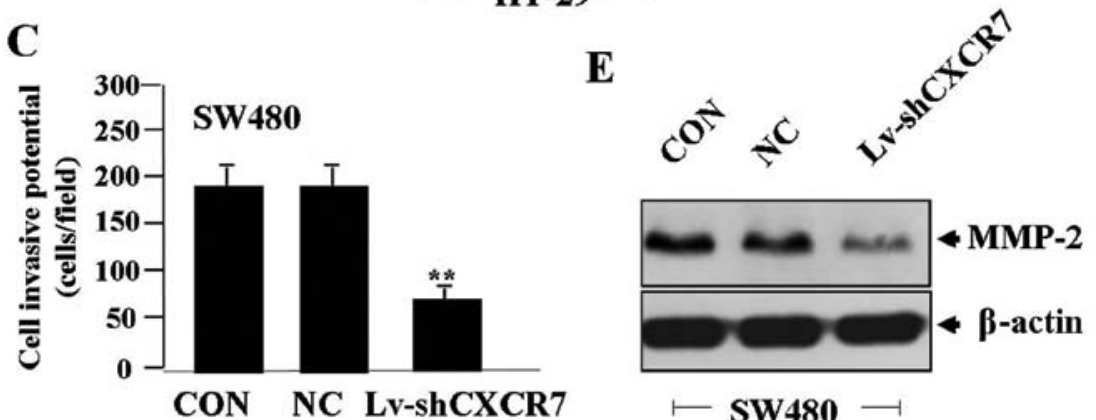

D

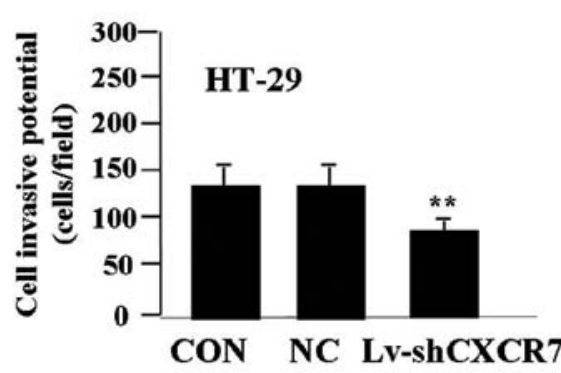

F
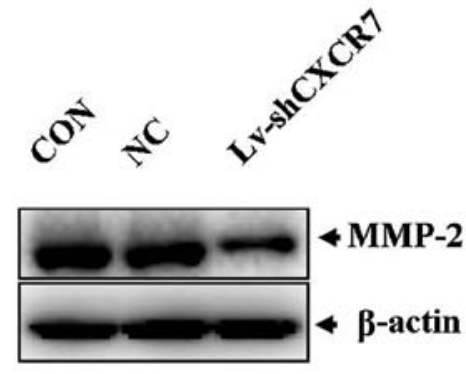

$\vdash$ HT-29 $\longrightarrow$

Figure 4. Effect of CXCR7 silencing on cell invasion. (A-D) The effect of CXCR7 on cell invasion was observed by Transwell assay. The invasive potential of CRC cells was significantly reduced in Lv-shCXCR7 group compared with CON and NC groups $\left({ }^{* *} \mathrm{P}<0.01\right)$. (E and F) The amount of MMP-2 protein was decreased in Lv-shCXCR7 group compared with CON and NC groups.

The effect of CXCR7 silencing on xenograft tumor growth. We investigated the effect of CXCR7 silencing on xenograft tumor growth in vivo. The mean volume of tumors in the experimental mice before treatment was $76.75 \pm 18.25 \mathrm{~mm}^{3}$. During the whole tumor growth period, the tumor growth activity was measured. The tumors treated with Lv-shCXCR7 grew substantially slower compared to the NC and PBS groups (Fig. 6A and B). When the tumors were harvested, the average weight and volume of the tumors in $\mathrm{Lv}$-shCXCR7 group were significantly smaller than those of the NC and PBS groups (Fig. 6C and D), suggesting that CXCR7 silencing was able to suppress growth of colon cancer cells.

\section{Discussion}

CXCR7, a member belonging to the membrane-bound $\mathrm{G}$ protein-coupled receptor superfamily, plays a role in cell growth, metastasis and angiogenesis of certain malignant tumors. CXCR7 is detected in all tumor samples from patients with neurilemmoma and meningioma (18), and upregulated in primary bone tumors with lung metastases (19). CXCR7 expression is correlated with solid tumor size and degree of differentiation, suggesting a potential function in tumor progression and differentiation (20). Therefore, we examined the expression of CXCR7 in CRC and analyzed the correlation of CXCR7 expression with clinicopathologic characteristics of CRC patients. It was found that CXCR7 expression, mainly localized in the cytoplasm and membrane, was significantly upregulated in CRC tissues compared with ANCT, and closely associated with Dukes staging and tumor invasion, indicating that CXCR7 might be implicated in the development of CRC. Some other studies show that CXCR7 correlates with unfavorable survival and prognosis of patients with renal cell carcinoma (5). Therefore, CXCR7 may provide a promising biomarker for detection and diagnosis of cancer (15).

Furthermore, the function of CXCR-7 in cancer need be explored. Some studies have shown that suppression of the expression or the activities of CXC-4/-7/-12 could remarkably 


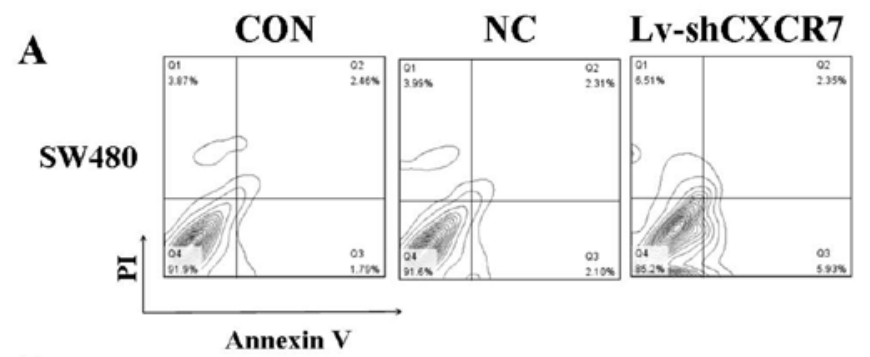

B

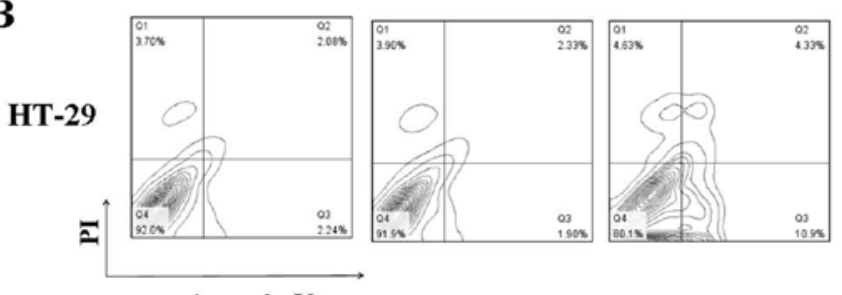

C

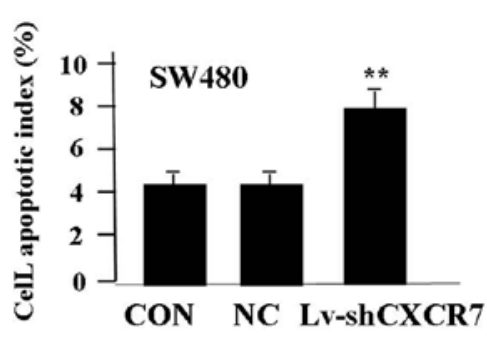

D

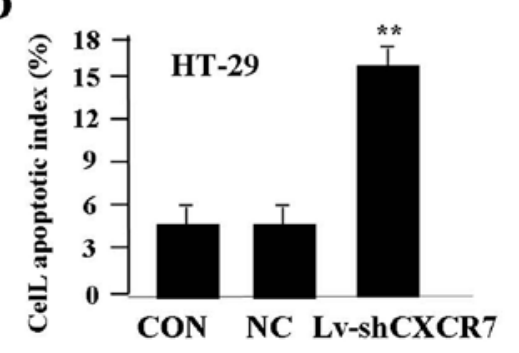

$\mathbf{E}$

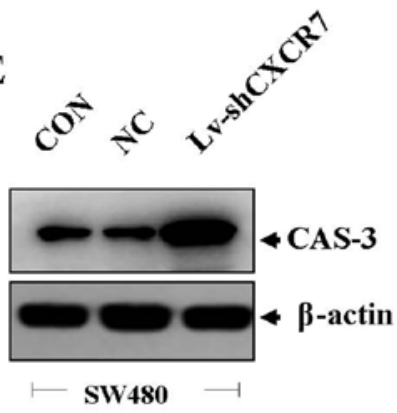

F

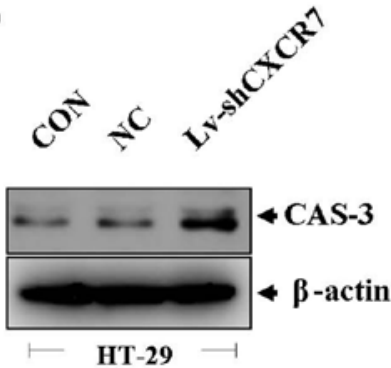

Figure 5. Effect of CXCR7 silencing on cell apoptosis. (A-D) Apoptotic indexes of CRC cells were much higher in Lv-shCXCR7 group than those in CON and NC groups $\left({ }^{* *} \mathrm{P}<0.01\right)$. (E and $\mathrm{F}$ ) The amount of CAS-3 protein was increased in $\mathrm{Lv}$-shCXCR7 group compared with CON and NC groups.
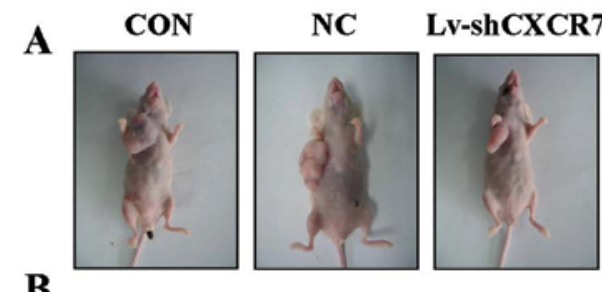

B

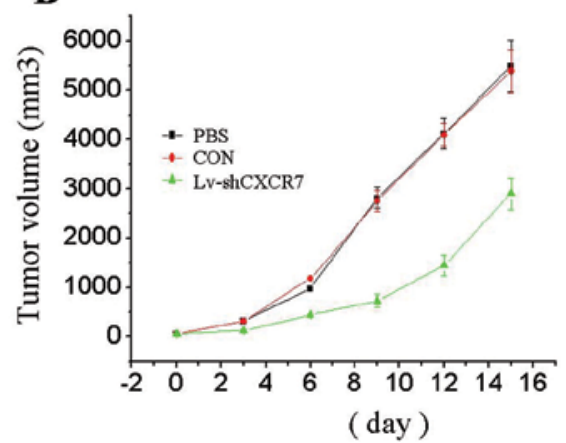

C

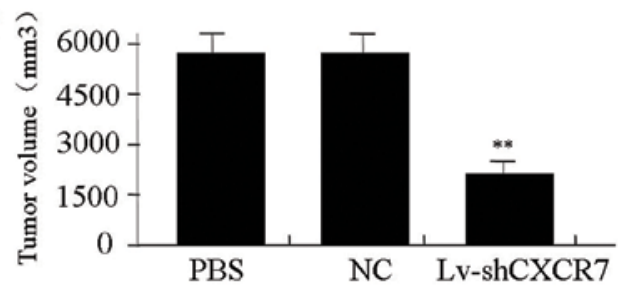

D

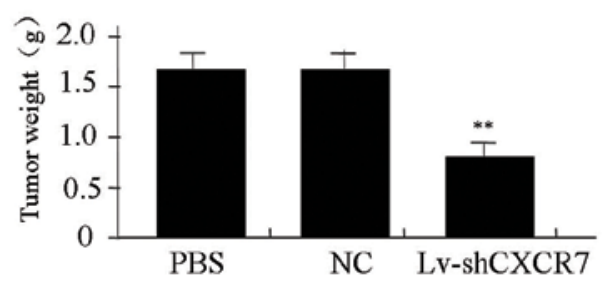

Figure 6. The effect of Lv-shCXCR7 on SW480 xenograft tumor growth. (A and B) During the whole tumor growth period, the tumor growth activity was measured. SW480 xenograft tumors treated with Lv-shCXCR7 grew substantially slower compared to those in NC and PBS groups. (C and D) The average weight and volume of the tumors in $\mathrm{Lv}$-shCXCR7 group were significantly smaller than those of theNC and PBS groups $\left.{ }^{(* *} \mathrm{P}<0.01\right)$. 
decrease cell progression, metastasis and angiogenesis in pancreatic cancer and brain tumors $(18,21)$. Downregulation of CXCR7 expression limits trans-endothelial migration of cancer cells into blood circulation, inhibits lymph node metastases and participates in the treatment of metastatic disease $(22,23)$, suggesting that CXCR7 may be a potential molecular target for use in carcinomatous therapy. However, some reports reveal the antitumor effects of CXCR7 through inhibition of tumor growth in hepatocellular carcinoma (24). Co-expression of CXCR7 and CXCR4 can reduce invasion and metastases of glioma cells (25). To manifest the function of CXCR7 in cancer, in our present study, it was found that silencing of CXCR7 gene repressed growth and invasion, and induced apoptosis in CRC cells. Likewise, the results of Guillemot et al support our study and show that targeting the CXCR7 axis blocks lung metastases from CRC (26), suggesting that CXCR7 might be a critical therapeutic target for the treatment of cancer.

Referring to the regulatory mechanisms of CXCR7 in cancer, a few studies have shown that CXCR7 can activate $\mathrm{G}$ protein pathways depending on SDF-1 (13), ERK1/2 and $\beta$-arrestin (27) involving tumorigenesis of human glioma. CXCL12-CXCR7 axis may directly activate the ERK1/2 signaling pathway in cancer cells, which can be blocked by deleting the carboxyl terminal domain of CXCR7 (28). However, few reports show that overexpression of CXCR7 markedly inhibits tumor proliferation and metastasis via downregulation of ERK and MMP-2/-9 expression (29). Our present findings indicated that knockdown of CXCR7 could downregulate the expression of $\mathrm{p}$-ERK and $\beta$-arrestin in CRC cells, suggesting that CXCR7 might be involved in the progression of CRC via regulation of $\mathrm{p}$-ERK and $\beta$-arrestin pathways.

PCNA is a nuclear protein that is expressed in proliferating cells and may be required for maintaining cell proliferation, used as a marker for cell proliferation of colon cancer (30). MMP-2 is thought to be a key enzyme involved in the degradation of type IV collagen and high level of MMP-2 in tissues is associated with tumor invasion and metastasis (31). CAS-3 is a member of the cysteine protease family, and plays a crucial role in apoptotic pathways by cleaving a variety of key cellular proteins (32). It has been reported that ERK $1 / 2$ and $\beta$-arrestin pathways promote growth and invasion of tumor cells via regulation of PCNA and MMP-2 expression (33). In our study, it was found that knockdown of CXCR7 downregulated the expression of PCNA and MMP-2, but upregulated CAS-3 expression in CRC cells, suggesting that CXCR7 might be implicated in the progression of CRC through ERK1/2 and $\beta$-arrestin pathways-mediated regulation of PCNA, MMP-2 and CAS-3 expression.

In conclusion, upregulation of CXCR7 expression is associated with tumor invasion, and silencing of CXCR7 gene represses the development of CRC cells through the ERK and $\beta$-arrestin pathway, suggesting that CXCR7 may serve as a potential therapeutic target for the treatment of CRC.

\section{References}

1. Wani HA, Beigh MA, Amin S, et al: Methylation profile of promoter region of p16 gene in colorectal cancer patients of Kashmir valley. J Biol Regul Homeost Agents 27: 297-307, 2013.
2. Kheirelseid EA, Miller N, Chang KH, et al: Clinical applications of gene expression in colorectal cancer. J Gastrointest Oncol 4: 144-57, 2013

3. Liao YX,Zhou CH,Zeng H, etal: The role of the CXCL12-CXCR4/ CXCR7 axis in the progression and metastasis of bone sarcomas (Review). Int J Mol Med 32: 1239-1246, 2013.

4. Ratajczak MZ, Kim C, Janowska-Wieczorek A, et al: The expanding family of bone marrow homing factors for hematopoietic stem cells: stromal derived factor 1 is not the only player in the game. Sci World J 2012: 758512, 2012.

5. Wang L, Chen W, Gao L, et al: High expression of CXCR4, CXCR7 and SDF-1 predicts poor survival in renal cell carcinoma. World J Surg Oncol 10: 212, 2012.

6. Monnier J, Boissan M, L'Helgoualc'h A, et al: CXCR7 is up-regulated in human and murine hepatocellular carcinoma and is specifically expressed by endothelial cells. Eur J Cancer 48: 138-148, 2012.

7. Hattermann K, Held-Feindt J, Lucius R, et al: The chemokine receptor CXCR7 is highly expressed in human glioma cells and mediates antiapoptotic effects. Cancer Res 70: 3299-3308, 2010.

8. Gahan JC, Gosalbez M, Yates T, et al: Chemokine and chemokine receptor expression in kidney tumors: molecular profiling of histological subtypes and association with metastasis. J Urol 187: 827-833, 2012

9. Liu Z, Sun DX, Teng XY, et al: Expression of stromal cellderived factor 1 and CXCR7 in papillary thyroid carcinoma. Endocr Pathol 23: 247-253, 2012.

10. Ehrlich A, Ray P, Luker KE, et al: Allosteric peptide regulators of chemokine receptors CXCR4 and CXCR7. Biochem Pharmacol 86: $1263-1271,2013$.

11. Hattermann $\mathrm{K}$ and Mentlein R: An infernal trio: the chemokine CXCL12 and its receptors CXCR4 and CXCR7 in tumor biology. Ann Anat 195: 103-110, 2013.

12. Schrevel M, Karim R, ter Haar NT, et al: CXCR7 expression is associated with disease-free and disease-specific survival in cervical CXCR7 patients. Br J Cancer 106: 1520-1525, 2012.

13. Odemis V, Lipfert J, Kraft R, et al: The presumed atypical chemokine receptor CXCR7 signals through $\mathrm{G}(\mathrm{i} / \mathrm{o})$ proteins in primary rodent astrocytes and human glioma cells. Glia 60: 372-381, 2012.

14. Wijtmans M, Maussang D, Sirci F, et al: Synthesis, modeling and functional activity of substituted styrene-amides as smallmolecule CXCR7 agonists. Eur J Med Chem 51: 184-192, 2012.

15. Maussang D, Mujić-Delić A, Descamps FJ, et al: Llamaderived single variable domains (nanobodies) directed against chemokine receptor CXCR7 reduce head and neck cancer cell growth in vivo. J Biol Chem 288: 29562-29572, 2013.

16. McGinn OJ, Marinov G, Sawan S, et al: CXCL12 receptor preference, signal transduction, biological response and the expression of 5T4 oncofoetal glycoprotein. J Cell Sci 125: 5467-5478, 2012

17. Hao M, Zheng J, Hou K, et al: Role of chemokine receptor CXCR7 in bladder cancer progression. Biochem Pharmacol 84: 204-214, 2012.

18. Tang T, Xia QJ, Chen JB, et al: Expression of the CXCL12/SDF-1 chemokine receptor CXCR7 in human brain tumours. Asian Pac J Cancer Prev 13: 5281- 5286, 2012.

19. Goguet-Surmenian E, Richard-Fiardo P, Guillemot E, et al: CXCR7-mediated progression of osteosarcoma in the lungs. $\mathrm{Br}$ J Cancer 109: 1579-1585, 2013.

20. Gebauer F, Tachezy M, Effenberger K, et al: Prognostic impact of CXCR4 and CXCR7 expression in pancreatic adenocarcinoma. J Surg Oncol 104: 140-145, 2011.

21. Guo JC, Li J, Yang YC, et al: Oligonucleotide microarray identifies genes differentially expressed during tumorigenesis of DMBA-induced pancreatic cancer in rats. PLoS One 8: e82910, 2013.

22. Baumhoer D, Smida J, Zillmer S, et al: Strong expression of CXCL12 is associated with a favorable outcome in osteosarcoma. Mod Pathol 25: 522-528, 2012.

23. Zabel BA, Lewén S, Berahovich RD, et al: The novel chemokine receptor CXCR7 regulates trans-endothelial migration of cancer cells. Mol Cancer 10: 73, 2011.

24. Zheng K, Li HY, Su XL, et al: Chemokine receptor CXCR7 regulates the invasion, angiogenesis and tumor growth of human hepatocellular carcinoma cells. J Exp Clin Cancer Res 29: 31, 2010.

25. Hattermann K, Mentlein R and Held-Feindt J: CXCL12 mediates apoptosis resistance in rat C6 glioma cells. Oncol Rep 27: 1348-1352, 2012. 
26. Guillemot E, Karimdjee-Soilihi B, Pradelli E, et al: CXCR7 receptors facilitate the progression of colon carcinoma within lung not within liver. Br J Cancer 107: 1944-1949, 2012.

27. Heinrich EL, Lee W, Lu J, et al: Chemokine CXCL12 activates dual CXCR4 and CXCR7-mediated signaling pathways in pancreatic cancer cells J Transl Med 10: 68, 2012.

28. Ray P, Mihalko LA, Coggins NL, et al: Carboxy-terminus of CXCR7 regulates receptor localization and function. Int J Biochem Cell Biol 44: 669-678, 2012.

29. Liu L, Zhao X, Zhu X, et al: Decreased expression of miR-430 promotes the development of bladder cancer via the upregulation of CXCR7. Mol Med Rep 8: 140-146, 2013.

30. Risio M: Cell proliferation in colorectal tumor progression: an immunohistochemical approach to intermediate biomarkers. J Cell Biochem (Suppl) 16G: 79-87, 1992.
31. Liabakk NB, Talbot I, Smith RA, et al: Matrix metalloprotease 2 (MMP-2) and matrix metalloprotease 9 (MMP-9) type IV collagenases in colorectal cancer. Cancer Res 56: 190-196, 1996.

32. Devarajan E, Sahin AA, Chen JS, et al: Downregulation of caspase 3 in breast cancer: a possible mechanism for chemoresistance. Oncogene 21: 8843-8851, 2002.

33. Dong S, Kong J, Kong F, et al: Insufficient radiofrequency ablation promotes epithelial-mesenchymal transition of hepatocellular carcinoma cells through Akt and ERK signaling pathways. J Transl Med 11: 273, 2013. 